Pacific

Journal of

Mathematics

\title{
VECTOR BUNDLES \\ WITH NO INTERMEDIATE COHOMOLOGY \\ ON FANO THREEFOLDS OF TYPE $V_{22}$
}

ENRIQUE ARRONDO AND DANIELE FAENZI 


\title{
VECTOR BUNDLES WITH NO INTERMEDIATE COHOMOLOGY ON FANO THREEFOLDS OF TYPE $V_{22}$
}

\author{
ENRIQUE ARRONDO AND DANIELE FAENZI
}

\begin{abstract}
We classify rank-2 vector bundles with no intermediate cohomology on the general prime Fano threefold of index 1 and genus 12. The structure of their moduli spaces is given by means of a monad-theoretic resolution in terms of exceptional bundles.
\end{abstract}

\section{Introduction}

The study of vector bundles with no intermediate cohomology, also called arithmetically Cohen-Macaulay bundles (see Definition 2.1), has been taken up by several authors. The well-known splitting criterion for projective spaces showed by Horrocks [1964] has been generalized by Ottaviani [1987; 1989] to Grassmannians and quadrics. Knörrer [1987] proved that line bundles and spinor bundles are the only ACM bundles on quadrics, while Buchweitz, Greuel and Schreyer showed in [1987] that only projective spaces and quadrics admit a finite number of equivalence classes of ACM bundles, up to twists.

On the other hand, the problem of classifying ACM bundles on special classes of varieties has been studied in several papers. Arrondo and Costa [2000] took up the case of prime Fano threefolds of index 2, while Faenzi [2005] considered the case of the index-2 prime threefold $V_{5}$.

Madonna classified rank-2 ACM bundles on the quartic threefold [2000], and got a numerical classification [2002] of the invariants of these bundles on any prime Fano threefold $V_{2 g-2}$ of index 1 and genus $g$, with $2 \leq g \leq 12$ and $g \neq 11$. In particular, he conjectured that all the cases of this classification occur on every such threefold $V_{2 g-2}$.

For higher dimensional varieties, the case of $\mathbb{G}\left(\mathbb{P}^{1}, \mathbb{P}^{4}\right)$ has been studied in [Arrondo and Graña 1999].

MSC2000: primary 14J60; secondary 14F05, 14J30, 14J45, 14D20.

Keywords: Cohen-Macaulay modules, intermediate cohomology, Fano threefolds $V_{22}$, canonical and half-canonical curves.

The first author was partially supported by the MCYT project BFM2003-03971. The second author was partially supported by Italian MIUR funds and Azione Integrata Italia-Spagna HI00-128. 
In this paper we consider rank-2 ACM bundles on the general prime Fano threefold $X$ of index 1 and genus 12 (see Definition 2.3). Write the Chern classes of a sheaf $\mathscr{F}$ on $X$ as integers (see Section 2), and we denote by $\mathscr{F}_{c_{1}, c_{2}}$ a rank-2 sheaf $\mathscr{F}$ on $X$ with $c_{1}(\mathscr{F})=c_{1}$ and $c_{2}(\mathscr{F})=c_{2}$. The main result of this paper states:

Theorem. On the general $X$ as above, there exist the following vector bundles with no intermediate cohomology:

(1) The bundle $\mathscr{F}_{-1,1}$ associated to a line contained in $X$;

(2) The bundle $\mathscr{F}_{0,2}$ associated to a conic contained in $X$;

(3) The bundle $\mathscr{F}_{-1, d}(1)$ associated to an elliptic curve of degree $d$, with $7 \leq d \leq$ 14

(4) The bundle $\mathscr{F}_{0,4}(1)$ associated to a canonical curve of degree 26 and genus 14 contained in $X$;

(5) The bundle $\mathscr{F}_{-1,15}(2)$ associated to a half-canonical curve $C_{60}^{59}$ of degree 59 and genus 60 contained in $X$.

These are the only possible indecomposable vector bundles with no intermediate cohomology on X, up to isomorphism and twists by line bundles.

The moduli space of semistable vector bundles with no intermediate cohomology is generically smooth, of dimension equal to 2 in Case (2), 2d-14 in Case (3), 5 in Case (4), and 16 in Case (5).

This gives a complete classification of ACM rank-2 bundles on the general Fano threefold $X$, together with a description of their moduli spaces. The main tools for proving the theorem are the study of elliptic curves in $X$ and the resolution of the diagonal on $X \times X$ obtained in [Faenzi 2006].

The paper is structured as follows: In Section 2 we state basic definitions and review some known facts concerning the threefold $X$. We also recall for the reader's convenience the available descriptions of $X$, which we will use frequently.

In Section 3 we briefly consider lines and conics contained in $X$. We also give a monad-theoretic interpretation of the Hilbert scheme of lines and conics in $X$. In Sections 4 and 5 we take up the analysis of elliptic, canonical, and half-canonical curves in $X$ that give rise to vector bundles with no intermediate cohomology, proving their existence and describing their associated moduli spaces.

\section{Preliminaries}

Let $Y$ be a smooth projective threefold with $\operatorname{Pic}(Y) \simeq \mathbb{Z}=\left\langle\mathcal{O}_{Y}(1)\right\rangle$ and $\mathrm{H}^{1}(\mathscr{O}(t))=$ $\mathrm{H}^{2}(\mathrm{O}(t))=0$ for any $t \in \mathbb{Z}$. Following standard terminology, we have: 
Definition 2.1. Given a sheaf $\mathscr{F}$ over $Y$, we say that $\mathscr{F}$ is $A C M$ (arithmetically Cohen-Macaulay) if $\mathrm{H}^{p}(Y, \mathscr{F}(t))=0$ for all $t \in \mathbb{Z}$ and $0<p<3$. Equivalently, we say that $\mathscr{F}$ has no intermediate cohomology.

We denote the dual of a vector bundle $\mathscr{F}$ by $\mathscr{F}^{*}$, and recall that if $\mathscr{F}^{*}$ has rank 2 then $\mathscr{F}^{*} \simeq \mathscr{F}\left(-c_{1}(\mathscr{F})\right)$.

We now review the Hartshorne-Serre correspondence between codimension-2 subvarieties and rank-2 vector bundles, originally introduced in [Serre 1963] and later considered by many authors; see for example [Hartshorne 1974; Vogelaar 1978; Okonek et al. 1980].

Definition 2.2. A complete subvariety $Z$ of $Y$ is called subcanonical if there exists a line bundle $\mathcal{O}(r)$ on $Y$ such that $\left.\mathscr{O}(r)\right|_{Z} \cong \omega_{Z}$. Let $Z$ be a subcanonical locally complete intersection codimension-2 subvariety of $Y$. By [Okonek et al. 1980, Theorem 5.1.1], there exist a rank-2 vector bundle $\mathscr{F}_{Z}$ over $Y$ and a section $s_{Z} \in$ $\mathrm{H}^{0}\left(Y, \mathscr{F}_{Z}^{*}\right)$ such that $Z=\left\{s_{Z}=0\right\}$, that is, $Z$ is the zero locus of $s_{Z}$. We say in this case that $\mathscr{F}_{Z}$ is associated to $Z$. We denote by $N_{Z, Y}$ the normal bundle of $Z$ in $Y$ and by $J_{Z, Y}$ the ideal sheaf of $Z$ in $Y$.

Under these hypotheses, we have the fundamental exact sequence

$$
0 \longrightarrow \operatorname{det} \mathscr{F}_{Z} \longrightarrow \mathscr{F}_{Z} \longrightarrow J_{Z, Y} \longrightarrow 0
$$

and the adjunction isomorphism

$$
\left.\left(\mathscr{F}_{Z}^{*}\right)\right|_{Z} \simeq N_{Z, Y}
$$

Definition 2.3. A prime Fano threefold of index 1 and genus 12 is a 3-dimensional algebraic variety $X$ with $\operatorname{Pic}(X) \simeq \mathbb{Z}=\left\langle O_{X}(1)\right\rangle$ and $\omega_{X} \cong O_{X}(-1)$, and with $\operatorname{deg} O_{X}(1)=22$. Any such $X$ is rational. We have $\mathrm{h}^{0}\left(\mathrm{O}_{X}(1)\right)=14$, while $\mathrm{CH}^{i}(X)$, the $i$-th Chow group of $X$, is isomorphic to $\mathbb{Z}$ for $i=1,2,3$.

From now on, $X$ will denote a prime Fano threefold of index 1 and genus 12 . We denote the Chern classes of a sheaf $\mathscr{F}$ on $X$ by integers $c_{i} \in \mathbb{Z}$, meaning that $c_{i}(\mathscr{F})=c_{i} \xi_{i}$, where $\xi_{i}$ is the generator of $\mathrm{CH}^{i}(X) \simeq \mathbb{Z}$ for $i=1,2,3$. Recall that $\xi_{2}$ is the class of a line in $X$.

Further, we define $\mu(\mathscr{F})$ as the rational number $c_{1}(\mathscr{F}) / \mathrm{rk} \mathscr{F}$. We say that a vector bundle $\mathscr{F}$ is normalized if $-\mathrm{rk} \mathscr{F}<c_{1}(\mathscr{F}) \leq 0$. Equivalently, $\mathscr{F}$ is normalized if $-1<\mu(\mathscr{F}) \leq 0$. Clearly, $\mu\left(\mathscr{F}_{1} \otimes \mathscr{F}_{2}\right)=\mu\left(\mathscr{F}_{1}\right)+\mu\left(\mathscr{F}_{2}\right)$.

We refer to [Huybrechts and Lehn 1997] for the definition of (semi)stability (in the sense of Mumford and Takemoto). A stable bundle $\mathscr{F}_{F}$ with $\mu(\mathscr{F})<0$ satisfies $\mathrm{h}^{0}(\mathscr{F})=0$. Recall that, by Hoppe's criterion, since $\operatorname{Pic}(X)$ is generated by $\mathrm{O}_{X}(1)$, a rank-2 bundle $\mathscr{F}$ on $X$ is stable if $\mathrm{h}^{0}(\mathscr{F}(t))=0$, for the only integer $t$ such that $c_{1}(\mathscr{F}(t))=0$ or $c_{1}(\mathscr{F}(t))=-1$. See for example [Okonek et al. 1980, Lemma 1.2.5]. 
From the Hirzebruch-Riemann-Roch formula for a vector bundle on $X$ of rank $r$ and Chern classes $c_{i}$, we obtain:

$$
\begin{aligned}
6 \chi(\mathscr{F}(s))=22 s^{3} r+11 s^{2}\left(3 r+6 c_{1}\right) & +s\left(23 r+66 c_{1}-6 c_{2}+66 c_{1}^{2}\right) \\
+6 r & +23 c_{1}-3 c_{2}-3 c_{1} c_{2}+33 c_{1}^{2}+3 c_{3}+22 c_{1}^{3} .
\end{aligned}
$$

Given a smooth projective variety $Y$ equipped with a very ample line bundle $\mathrm{O}_{Y}(1)$, for any integer $r$ and string $c$ with $c_{i} \in \mathrm{CH}^{i}(Y)$ (identified with integers whenever possible), we write $\mathrm{M}_{Y}(r ; c)$ for the moduli space of rank-r semistable sheaves on $Y$ with Chern classes $c_{i}$.

By virtue of the exact sequence (1), the Hilbert polynomial and the Chern classes of $\mathscr{F}_{Z}$ are determined by the Hilbert polynomial of $Z$. Denote by $P_{[Z]}$ the Hilbert polynomial of $Z$ with respect to the polarization $O_{Y}(1)$, and by $\operatorname{Hilb}_{[Z]}(Y)$ the Hilbert scheme of closed subschemes of $Y$ with Hilbert polynomial $P_{[Z]}$ (see [Huybrechts and Lehn 1997, Page 41]). Further, if $Z$ is a curve of degree $d$ and genus $g$ contained in $X$, we denote $\operatorname{Hilb}_{[Z]}(X)$ by $\mathscr{H}_{d, g}(X)$.

If the bundle $\mathscr{F}_{Z}$ is stable, the Hartshorne-Serre correspondence provides a morphism

$$
\tau: \operatorname{Hilb}_{[Z]}(X) \rightarrow \mathrm{M}_{X}\left(2 ; c_{1}\left(\mathscr{F}_{Z}\right), c_{2}\left(\mathscr{F}_{Z}\right)\right), \quad[Z] \mapsto\left[\mathscr{F}_{Z}\right]
$$

We next recall some of the available constructions of the threefold $X$. We also sketch the description of four fundamental vector bundles $E, U, Q, K$ of respective ranks 2, 3, 4, 5 and defined over $X$.

We refer to [Mukai 1992, 2004; Schreyer 2001; Faenzi 2006] for proofs and more details.

Nets of dual quadrics and twisted cubics. Let $k$ be an algebraically closed field. Let $A \simeq k^{4}$ and $B \simeq k^{3}$ be $k$-vector spaces, and let $R(A)=k[A]$ and $R(B)=k[B]$ be polynomial algebras. Let $\mathrm{S}^{d} A=R(A)_{d}$ be the $d$-th symmetric power of the vector space $A$.

Given a twisted cubic $\Gamma$, we have $P_{[\Gamma]}(t)=\chi\left(\bigcirc_{\Gamma}(t)\right)=3 t+1$. We consider the Hilbert scheme $\mathrm{Hilb}_{3 t+1}(\mathbb{P}(A))$ of closed subschemes of $\mathbb{P}(A)$ with Hilbert polynomial $3 t+1$, and we define the variety $\mathrm{H}$ to be the irreducible component of Hilb $_{3 t+1}(\mathbb{P}(A))$ containing the rational normal cubics in $\mathbb{P}(A)$, as constructed in [Ellingsrud et al. 1987]. Given a twisted cubic $[\Gamma] \in \mathrm{H}$, we denote by $J_{\Gamma}$ the ideal sheaf of $\Gamma$ in $\mathbb{P}(A)$. The open subset $\mathrm{H}_{c}$ consisting of points that are arithmetically Cohen-Macaulay embeds in $\mathbb{G}\left(k^{3}, \mathrm{~S}^{2} A\right)$ by means of the vector bundle $U_{\mathrm{H}}$ whose fiber over $[\Gamma] \in \mathrm{H}_{c}$ is $\operatorname{Tor}_{1}^{R[A]}\left(R[A] / J_{\Gamma}, k\right)_{2} \simeq k^{3}$. Equivalently, we associate to any $[\Gamma] \in \mathrm{H}$ the net of quadrics in $\mathbb{P}(A)$ vanishing on $\Gamma$. 
Definition 2.4. A net of dual quadrics $\Psi$ (parametrized by $B$ ) in $\mathbb{P}(A)$ is defined as a surjective map $\Psi: \mathrm{S}^{2} A \rightarrow B$. Let $V_{\Psi}=\operatorname{ker} \Psi$. Given a general net $\Psi$, we define:

$$
\begin{aligned}
X_{\Psi} & =\left\{[\Gamma] \in \mathrm{H} \subset \operatorname{Hilb}_{3 t+1}\left(\mathbb{P}^{3}\right) \mid \Psi\left(\mathrm{H}^{0}\left(J_{\Gamma}(2)\right)\right)=0\right\} \\
& =\left\{[\Gamma] \in \mathrm{H} \subset \mathrm{Hilb}_{3 t+1}\left(\mathbb{P}^{3}\right) \mid \mathrm{H}^{0}\left(J_{\Gamma}(2)\right) \subset V_{\Psi}\right\} .
\end{aligned}
$$

We define the bundle $U$ on $X=X_{\Psi}$ as the restriction of $U_{\mathrm{H}}$ to $X$.

Definition 2.5. Let $\Psi$ be a general net of dual quadrics and $X=X_{\Psi}$. There is a rank-2 vector bundle $E$ on $X$ defined by $E_{[\Gamma]}=\operatorname{Tor}_{2}^{R[A]}\left(R[A] / J_{\Gamma}, k\right)_{3} \simeq k^{2}$. Equivalently, we associate to any $[\Gamma] \in \mathrm{H}$ its space of first-order syzygies.

Lemma 2.6 [Faenzi 2006, Lemma 6.3]. The bundle $E^{*}$ is globally-generated and $A C M$, with $\mathrm{h}^{0}\left(E^{*}\right)=8$. Consider the rank-6 bundle $E^{\prime}=\operatorname{ker}\left(\mathrm{H}^{0}\left(E^{*}\right) \otimes 0 \rightarrow E^{*}\right)$. The bundle $E^{\prime}$ is also stable and ACM.

Plane quartics. Let $B$ be a 3-dimensional $k$-vector space and $F \in \mathrm{S}^{4} B$ a plane quartic. Set $\check{\mathbb{P}}^{2}=\mathbb{P}\left(B^{*}\right)$. Take the Hilbert scheme $\mathrm{Hilb}_{6}\left(\check{\mathrm{P}}^{2}\right)$ of zero-dimensional length 6 closed subschemes of $\check{\mathbb{P}}^{2}$. Define the subvariety of $\operatorname{Hilb}_{6}\left(\check{\mathbb{P}}^{2}\right)$ consisting of polar hexagons to $F$,

$$
X_{F}=\left\{\Lambda=\left(f_{1}, \ldots, f_{6}\right) \in \operatorname{Hilb}_{6}\left(\check{\mathbb{P}}^{2}\right) \mid f_{1}^{4}+\cdots+f_{6}^{4}=F\right\} .
$$

Lemma 2.7 [Mukai 2004; Schreyer 2001]. For a general $F$, the variety $X_{F}$ is a prime Fano threefold of index 1 and genus 12. Given a net of dual quadrics $\Psi$, there exists a quartic form $F$ such that $X_{F} \simeq X_{\Psi}$.

Definition 2.8. Let $F$ be a general plane quartic and let $X=X_{F}$. There is a rank-5 vector bundle $K$ on $X_{F}$ defined over an element $\Lambda=\left(f_{1}, \ldots, f_{6}\right) \in X_{F}$ by $K_{\Lambda}=\left\langle f_{1}^{4}, \ldots, f_{6}^{4}\right\rangle / F$. The bundle $K^{*}$ is stable and ACM, with $\mathrm{h}^{0}\left(K^{*}\right)=14$ and $c_{1}(K)=-2$ [Faenzi 2006, Lemma 6.1 and 6.2].

Remark 2.9. Under the hypothesis of Lemma 2.7, there is a natural isomorphism $V_{\Psi} \simeq \mathrm{S}^{3} B / F\left(B^{*}\right)$, where we consider $F$ as a map $B^{*} \rightarrow \mathrm{S}^{3} B$ taking an element $\partial \in B^{*}$ to the cubic form $\partial(F)$ (apolarity action). We set $V_{F}=S^{3} B / F\left(B^{*}\right)$. The fiber of $U$ over an element $\Lambda=\left(f_{1}, \ldots, f_{6}\right) \in X_{F}$ is naturally identified with $\left\langle f_{1}^{3}, \ldots, f_{3}^{4}\right\rangle / F\left(B^{*}\right)$. The global sections of $U^{*}$ and $K^{*}$ are then identified with $V_{F}=S^{3} B / F\left(B^{*}\right)$ and $S^{4} B / F$, respectively. An element $\partial$ of $B^{*}$ gives a map $\mathrm{S}^{4} B \rightarrow \mathrm{S}^{3} B$ by the apolarity action and, therefore, a homomorphism $\partial: K \rightarrow U$.

Nets of alternating 2-forms. Let $V$ be a 7-dimensional $k$-vector space and $B$ a 3dimensional one. Let $\mathrm{G}$ be the Grassmannian $\mathbb{G}\left(k^{3}, V\right)$. Define $U_{\mathrm{G}}$ as the universal rank-3 subbundle, and $Q_{\mathrm{G}}$ as the universal rank-4 quotient bundle on $\mathrm{G}$. Let $\sigma$ 
be a section of $B^{*} \otimes \bigwedge^{2} U_{G}^{*}$. Equivalently, $\sigma$ is a net of alternating 2-forms $\sigma \in$ $B^{*} \otimes \bigwedge^{2} V^{*}$.

Definition 2.10. Define $X_{\sigma}$ as the zero locus in $\mathrm{G}$ of $\sigma \in B^{*} \otimes \wedge^{2} V^{*}$. For a general $\sigma$, the variety $X_{\sigma}$ is a prime Fano threefold of index 1 and genus 12 .

Lemma 2.11 [Mukai 2004]. Given a general plane quartic $F$, there is a net of alternating 2-forms $\sigma_{F}$ such that $X_{\sigma} \simeq X_{F}$.

From now on, we identify $X$ with $X_{\Psi} \simeq X_{F} \simeq X_{\sigma}$, where $\Psi$ is a general net of dual quadrics, $F$ is the quartic form provided by Lemma 2.7, and $\sigma$ is the net of alternating 2-forms given by Lemma 2.11. In particular, we fix the 3- and 4-dimensional $k$-vector spaces $B$ and $A$. Recall that, by Remark 2.9, we have $V \simeq V_{F} \simeq V_{\Psi}$. We also notice that, under our hypotheses, $\left.\left.\left(U_{\mathrm{G}}\right)\right|_{X} \simeq\left(U_{\mathrm{H}}\right)\right|_{X}$. Thus, we denote also by $U$ the restriction of the vector bundle $U_{\mathrm{G}}$ to $X_{\sigma}$. We set $Q=\left.\left(Q_{\mathrm{G}}\right)\right|_{X}$.

Lemma 2.12. There are natural isomorphisms

$$
\begin{array}{ll}
\operatorname{Hom}\left(U, Q^{*}\right) \simeq B, & \operatorname{Hom}(E, U) \simeq A^{*}, \\
\operatorname{Hom}(K, U) \simeq B^{*}, & \operatorname{Hom}(E, K) \simeq A .
\end{array}
$$

Moreover, there are exact sequences

$$
\begin{aligned}
& 0 \longrightarrow U \longrightarrow V \otimes \mathcal{O} \longrightarrow Q \longrightarrow 0, \\
& 0 \longrightarrow K \longrightarrow B \otimes U \longrightarrow Q^{*} \longrightarrow 0, \\
& 0 \longrightarrow \wedge^{2} U \longrightarrow A \otimes E \longrightarrow K \longrightarrow 0 \\
& 0 \longrightarrow E \longrightarrow 0^{\oplus 8} \longrightarrow\left(E^{\prime}\right)^{*} \longrightarrow 0 .
\end{aligned}
$$

The Chern classes of these bundles are

$$
\begin{array}{lll}
c_{1}(E)=-1, & c_{2}(E)=7, & \\
c_{1}(U)=-1, & c_{2}(U)=10, & c_{3}(U)=-2, \\
c_{1}\left(Q^{*}\right)=-1, & c_{2}\left(Q^{*}\right)=12, & c_{3}\left(Q^{*}\right)=-4, \\
c_{1}(K)=-2, & c_{2}(K)=40, & c_{3}(K)=-20, \\
c_{1}\left(E^{\prime}\right)=-1, & c_{2}\left(E^{\prime}\right)=15, & c_{3}\left(E^{\prime}\right)=-8 .
\end{array}
$$

Proof. The exact sequences (6) and (7), together with (4) and the first isomorphism in (5), are proved in [Faenzi 2006, Lemma 6.1]. The sequence (8) follows from [Faenzi 2006, Proposition 6.4], while (9) is Lemma 2.6. The second isomorphism in (5) follows from [Faenzi 2006, Corollary 6.8]. The Chern classes of $U, Q^{*}$ and $\wedge^{2} U$ are easily computed by restriction from $\mathbb{G}\left(k^{3}, V\right)$. Finally, the Chern classes of $K, E$ and $E^{\prime}$ follow from the exact sequences (7), (8) and (9). 
Birational geometry. We briefly sketch the birational geometry of $X$ following [Iskovskih 1978, 1989]. Fano's double projection from a line is used, and we refer to [Iskovskikh and Prokhorov 1999] for a complete treatment.

Let $V_{5}$ be the del Pezzo threefold obtained by cutting $\mathbb{G}\left(\mathbb{P}^{1}, \mathbb{P}^{4}\right) \subset \mathbb{P}^{9}$ with a general $\mathbb{P}^{6} \subset \mathbb{P}^{9}$. Denote by $S_{5}$ a general hyperplane section of $V_{5}$.

It turns out that our $X$ is birational to $V_{5}$ under the double projection from a line contained in $X$. We will use this map to embed in $X$ some elliptic curves contained in $V_{5}$.

The divisor $S_{5}$ is a degree 5 del Pezzo surface, hence it is isomorphic to the blow up of $\mathbb{P}^{2}$ at 4 points $B_{1}, \ldots, B_{4}$. Further, we have $\omega_{S_{5}}^{*} \simeq O_{S_{5}}(1) \simeq \mathcal{O}\left(3 \ell-\sum b_{i}\right)$, where $\ell$ is the class of a line in $\mathbb{P}^{2}$ and $b_{i}$ is the exceptional divisor over the point $B_{i}$.

Recall that, by [Iskovskikh and Prokhorov 1999], the threefold $V_{5}$ contains a rational normal curve $C_{0}^{5}$ of degree 5 (restrict to $S_{5}$ and take the divisor $2 \ell-b_{1}$ ). Furthermore, $C_{0}^{5}$ has exactly 3 chords $T_{1}, T_{2}, T_{3}$. Indeed, any chord of $C_{0}^{5}$ is contained in $S_{5}$, and the only lines in $S_{5}$ meeting $C_{0}^{5}$ in two points are of the form $\ell-b_{i}-b_{j}$ for $1<i<j$.

Denote by $H_{V_{5}}$ the divisor associated to $\mathcal{O}_{V_{5}}(1)$. The linear system $3 H_{V_{5}}-2 C_{0}^{5}$ defines a birational map $\varphi: V_{5} \rightarrow X$. Let $\tilde{X}$ be the variety obtained by blowing up $V_{5}$ along $C_{0}^{5}$ and then along the proper preimages of $T_{1}, T_{2}, T_{3}$. Denote by $\psi_{1}$ the contraction to $V_{5}$. There also exists a contraction $\psi_{2}: \tilde{X} \rightarrow X$, and we have $\varphi \circ \psi_{1}=\psi_{2}$.

Definition 2.13. Fix a general hyperplane section $S_{5}$ of $V_{5}$ and an isomorphism $S_{5} \rightarrow \mathrm{Bl}_{B_{1}, \ldots, B_{4}}\left(\mathbb{P}^{2}\right)$ (there is a finite number of such isomorphisms). Let $b_{i}$ be the exceptional divisors of $S_{5}$ over $B_{i}$. For a given rational normal curve $C_{0}^{5} \subset V_{5}$ with chords $\left\{T_{1}, T_{2}, T_{3}\right\}$, let $\left\{e_{1}, \ldots, e_{5}\right\}=S_{5} \cap C_{0}^{5}$ and $f_{i}=S_{5} \cap T_{i}$. On $S_{5}$, define $\mathscr{L}=9 \ell-3 \sum b_{i}-2 \sum e_{j}-\sum f_{k}$. We have $\left.\varphi\right|_{S_{5}}=\varphi_{|\mathscr{L}|}$, where $\varphi_{|\mathscr{L}|}$ is the map associated to the linear system $|\mathscr{L}|$.

Resolution of the diagonal. We recall here the resolution of the diagonal on $X$ and the induced Beilinson theorem. We refer to [Gorodentsev 1990; Rudakov 1990; Drezet 1986] for the general setup on exceptional collections and mutations.

Define the collection $\left(G_{3}, \ldots, G_{0}\right)=\left(E, U, Q^{*}, \mathcal{O}\right)$. This collection is strongly exceptional, that is, $\operatorname{Ext}^{p}\left(G_{j}, G_{i}\right)=0$ if $p>0$ or $i>j$, as proved in [Kuznetsov 1996]. Further, we define the collection $\left(G^{3}, \ldots, G^{0}\right)=(E, K, U, \mathcal{O})$. The following lemma, proved in [Faenzi 2006, Theorem 7.2], states that these two collections fit together to give a resolution of $\mathrm{O}_{\Delta}$ over $X \times X$.

Lemma 2.14. For a general $X$, there exists a resolution of $\mathrm{O}_{\Delta}$ on $X \times X$ of the form:

$$
0 \longrightarrow G_{3} \otimes G^{3} \longrightarrow \cdots \longrightarrow G_{0} \otimes G^{0} \longrightarrow \mathrm{O}_{\Delta} \longrightarrow 0 .
$$


Any coherent sheaf $\mathscr{F}$ on $X$ is functorially isomorphic to the cohomology of a complex $\mathscr{C}_{\text {FF }}$ whose terms are

$$
\mathscr{C}_{\mathscr{F}}^{k}=\bigoplus_{i-j=k} \mathrm{H}^{i}\left(\mathscr{F} \otimes G^{j}\right) \otimes G_{j} .
$$

Alternatively, $\mathscr{F}_{F}$ is functorially isomorphic to the cohomology of a complex $\mathscr{D}_{\mathscr{F}}$ whose terms are

$$
\mathscr{D}_{\mathscr{F}}^{k}=\bigoplus_{i-j=k} \mathrm{H}^{i}\left(\mathscr{F}_{F} \otimes G_{j}\right) \otimes G^{j} .
$$

The following Castelnuovo-Mumford regularity for the collection $\left(G_{3}, \ldots, G_{0}\right)$ is a consequence of Lemma 2.14; see [Faenzi 2006, Corollary 7.4].

Corollary 2.15. Let $\mathscr{F}$ be a coherent sheaf on $X$. If $\mathrm{H}^{p}\left(G_{p} \otimes \mathscr{F}\right)=0$ for $p>0$, then $\mathscr{F}$ is globally-generated.

Vector bundles with no intermediate cohomology. Recall from the introduction that a rank-2 vector bundle $\mathscr{F}$ with $c_{1}(\mathscr{F})=c_{1}$ and $c_{2}(\mathscr{F})=c_{2}$ is denoted by $\mathscr{F}_{c_{1}, c_{2}}$. Similarly, a curve of genus $g$ and degree $d$ is denoted by $C_{g}^{d}$.

Lemma 2.16 (Madonna). The only possible classes of indecomposable normalized rank-2 ACM vector bundles on $X$ are, up to isomorphism:

(1) the unstable bundle $\mathscr{F}_{-1,1}$ associated to a line in $X$;

(2) the semistable bundle $\mathscr{F}_{0,2}$ associated to a conic in $X$;

(3) the stable bundle $\mathscr{F}_{-1, d}(1)$ associated to an elliptic curve $C_{1}^{d}$ contained in $X$, with $7 \leq d \leq 14$;

(4) the stable bundle $\mathscr{F}_{0,4}(1)$ associated to a canonical curve $C_{14}^{26}$ in $X$;

(5) the stable bundle $\mathscr{F}_{-1,15}(2)$ associated to a half-canonical curve $C_{60}^{59}$ contained in $X$.

In each case, the smallest $t \in \mathbb{Z}$ with $\mathrm{h}^{0}(\mathscr{F}(t)) \neq 0$ is the one stated.

Proof. We refer to [Madonna 2002] for the full proof, with the exception of the condition $d \geq 7$ in (3), which we prove at the end of Section 4. We nonetheless sketch here the Madonna's main argument.

Considering the first twist $\mathscr{F}_{c_{1}, c_{2}}$ of $\mathscr{F}_{F}$ by a nonzero global section $s$, one proves easily that $Z=\{s=0\}$ is a connected curve of arithmetic genus $1+1 / 2\left(c_{1} c_{2}-c_{2}\right)$ and degree $c_{2}$. Therefore $c_{1} \geq 1-2 / c_{2} \geq-1$, so $\mathscr{F}$ is stable except for $c_{1}=-1$ or $c_{1}=0$, which correspond, respectively, to Cases (1) and (2).

For $c_{1}=1$, we end up in Case (3) and, making use of (1), it is easy to check that $d \leq 14$. 
For $c_{1}>1$, we find $\mathrm{h}^{p}\left(\mathscr{F}_{c_{1}, c_{2}}(-1)\right)=0$ and $\mathrm{h}^{p}\left(\mathscr{F}_{c_{1}, c_{2}}(-2)\right)=0$ for any $p$. Take the following polynomial equations in the variables $c_{1}$ and $c_{2}$ :

$$
\left\{\begin{array}{l}
\chi\left(\mathscr{F}_{c_{1}, c_{2}}(-1)\right)=0, \\
\chi\left(\mathscr{F}_{c_{1}, c_{2}}(-2)\right)=0 .
\end{array}\right.
$$

When $c_{1}>1$, we find Cases (4) and (5) as the only solutions.

\section{Lines and conics}

It is classically known that $X$ contains a one-dimensional family of lines and a two-dimensional family of smooth conics; see [Iskovskikh and Prokhorov 1999, Propositions 4.2.2 and 4.2.5] and references therein. Denote a line in $X$ by $C_{0}^{1}$ and a conic in $X$ by $C_{0}^{2}$. We will just provide resolutions of the sheaf ${ }_{C_{0}^{1}}(-1)$ and of the bundle $\mathscr{F}_{C_{0}^{2}}$ with respect to the collection $\left(G_{3}, \ldots, G_{0}\right)$. This will give a straightforward description of the Hilbert schemes of lines and conics in $X$.

Lemma 3.1. The sheaf $\mathscr{O}_{C_{0}^{1}}(-1)$ admits the resolution

$$
0 \longrightarrow E \longrightarrow K \stackrel{{ }^{\alpha} C_{0}^{1}}{\longrightarrow} U \longrightarrow{ }^{O_{C}^{1}}(-1) \longrightarrow 0 .
$$

The map $\alpha_{C_{0}^{1}} \in \operatorname{Hom}(K, U) \simeq B^{*}$ degenerates along a line $C_{0}^{1}$ if and only if it lies in the discriminant quartic curve $\operatorname{det} \Psi^{\top} \subset \check{\mathbb{P}}^{2}=\mathbb{P}\left(B^{*}\right)$. In particular, the Hilbert scheme of lines in $X$ is isomorphic to the curve $\operatorname{det} \Psi^{\top}$.

Proof. Clearly, we have $\left(G_{j}\right)_{C_{0}^{1}} \simeq \mathcal{O}_{\mathbb{P}^{1}}(-1) \oplus \mathcal{O}_{\mathbb{P} 1}^{4-j}$. Hence, $\mathrm{h}^{1}\left(G_{j} \otimes \mathcal{O}_{C_{0}^{1}}(-1)\right)=1$ for $j=1,2$, 3. By Lemma 2.14, the sheaf $O_{C_{0}^{1}}(-1)$ admits the resolution (10).

It is known from [Schreyer 2001, Theorem 6.1] that the Hilbert scheme of lines in $X$ is isomorphic to the curve $\operatorname{det} \Psi^{\top}$. We nonetheless sketch here a simpler argument.

From (5) follows the isomorphism $\operatorname{Hom}(K, U) \simeq B^{*}$. The application of the functor $\operatorname{Hom}(E,-)$ to a morphism $\alpha: K \rightarrow U$ corresponds, under the morphism $\Psi^{\top}: B^{*} \rightarrow S^{2} A^{*}$, to the linear map $\alpha \mapsto \Psi^{\top}(\alpha)$. That is, $\alpha$ is taken by $\operatorname{Hom}(E,-)$ to a linear map $\Psi^{\top}(\alpha): A \rightarrow A^{*}$. Since both $\operatorname{Hom}(E, K) \otimes E \rightarrow K$ and $\operatorname{Hom}(E, U) \otimes E \rightarrow U$ are epimorphisms, it follows that $\operatorname{Hom}(E, \alpha)$ is surjective if and only if $\alpha$ is surjective. This fails to hold precisely when $\alpha$ lies in the discriminant curve $\operatorname{det} \Psi^{\top}$, in which case there is a unique map $E \rightarrow \operatorname{ker} \alpha$. This map is an isomorphism. By a Hilbert polynomial computation, coker $\alpha$ is isomorphic to ${ }^{O_{C_{0}^{1}}}(-1)$.

Lemma 3.2 (Takeuchi). Through any point in $X$ there exists a finite number of conics contained in $X$. The Hilbert scheme of conics in $X$ is isomorphic to $\mathbb{P}(B)$. 
Proof. The first statement is proved in [Takeuchi 1989]. One may also consult [Iskovskikh and Prokhorov 1999, Lemma 4.2.6].

For any conic $C_{0}^{2}$ in $X$, there exists an exact sequence

$$
0 \longrightarrow U \longrightarrow Q^{*} \longrightarrow J_{C_{0}^{2}, X} \longrightarrow 0 .
$$

Any homomorphism $U \rightarrow Q^{*}$ degenerates along a conic. Since $\operatorname{Hom}\left(U, Q^{*}\right) \simeq B$, the lemma is proved.

Corollary 3.3. The set of stable points in the moduli space $\mathrm{M}_{X}(2 ; 0,2)$ is empty. The set of semistable points is isomorphic to $\mathbb{P}^{2}=\mathbb{P}(B)$. The bundle $\mathscr{F}_{0,2}$ of Lemma 2.16, Case (2), admits the resolution

$$
0 \longrightarrow U \longrightarrow Q^{*} \oplus \mathrm{O} \longrightarrow \mathscr{F}_{0,2} \longrightarrow 0 .
$$

Proof. Since the bundle $\mathscr{F}_{0,2}$ admits a unique global section $s$, and since $s$ vanishes along a conic $C_{0}^{2}$, there exists an isomorphism between $\mathrm{M}_{X}(2 ; 0,2)$ and $\operatorname{Hilb}_{2 t+1}(X) \simeq \mathbb{P}^{2}$, the Hilbert scheme of conics contained in $X$. The bundle $\mathscr{F}_{0,2}$ is strictly semistable for $c_{1}(\mathscr{F})=0$.

In this case, the exact sequence (1) reads

$$
0 \longrightarrow \mathrm{O} \longrightarrow \mathscr{F}_{0,2} \longrightarrow J_{C_{0}^{2}, X} \longrightarrow 0 .
$$

Since $\operatorname{Ext}^{1}\left(Q^{*}, \mathcal{O}\right)=0$, any morphism $Q^{*} \rightarrow J_{C_{0}^{2}, X}$ lifts to a morphism $Q^{*} \rightarrow$ $\mathscr{F}_{0,2}$. Considering the map $\mathcal{O} \rightarrow \mathscr{F}_{0,2}$ in the exact sequence (12) and lifting the projection $Q^{*} \rightarrow J_{C_{0}^{2}, X}$ in the exact sequence (11), we obtain a surjective bundle map $Q^{*} \oplus \mathcal{O} \rightarrow \mathscr{F}_{0,2}$ whose kernel is isomorphic to $U$. This provides the desired resolution.

\section{Elliptic curves}

In this section we prove the existence in $X$ of elliptic curves with the properties required by Case (3) of Lemma 2.16. In particular, the degree of these curves varies from 7 to 14 . The case $7 \leq d \leq 13$ is considered in Proposition 4.1, while the case $d=14$ is considered in Proposition 4.4. In the latter we also deal with the case $d=15$, which we will need in Section 5 .

Proposition 4.1. On the general variety $X$, there exist smooth elliptic curves $C_{1}^{d}$ of any degree $d$, for $7 \leq d \leq 13$. The curve $C_{1}^{d}$ is contained in exactly $14-d$ independent hyperplanes.

We will construct smooth elliptic curves in $X$ by means of the birational map $\varphi: V=V_{5} \rightarrow X$ of page 207.

Lemma 4.2. Let $S=S_{5}$ be a fixed hyperplane section of $V$, and fix the notations from page 207. The irreducible component $\mathrm{H}_{5 t+1}$ of the Hilbert scheme 
$\mathrm{Hilb}_{5 t+1}(V)$ containing smooth rational normal quintics in $V$ has dimension 10 at a general $\left[C_{0}^{5}\right]$. There is a dominant map $\zeta: \mathrm{H}_{5 t+1}(V) \rightarrow \mathrm{Hilb}_{5}\left(\mathbb{P}^{2}\right)$ defined by $\zeta:\left[C_{0}^{5}\right] \mapsto e_{1}+\cdots+e_{5}$.

Proof. Set $C=C_{0}^{5}$. First, notice that by the Riemann-Roch formula we have $\operatorname{expdim}\left(\mathscr{T}_{\mathrm{H}_{5 t+1}(V),[C]}\right)=10$, because $\operatorname{deg} N_{C, V}=10$ and so $\chi\left(N_{C, V}\right)=10$. Since $C \subset S$, we have the exact sequence of normal bundles

$$
\left.0 \longrightarrow N_{C, S} \longrightarrow N_{C, V} \longrightarrow\left(N_{S, V}\right)\right|_{C} \longrightarrow 0 \text {. }
$$

Now, by computing $\left(2 \ell-b_{1}\right)^{2}=3$, after the identification $C \simeq \mathbb{P}^{1}$ we get $N_{C, S} \simeq \mathrm{O}_{\mathbb{P}^{1}}(3)$ and obtain an exact sequence

$$
0 \longrightarrow \mathbb{O}_{\mathbb{P}^{1}}(3) \longrightarrow N_{C, V} \longrightarrow \mathrm{O}_{\mathbb{P}^{1}}(5) \longrightarrow 0 \text {. }
$$

Therefore $\mathrm{h}^{0}\left(N_{C, V}\right)=\chi\left(N_{C, V}\right)=10$, so $\mathrm{H}_{5 t+1}(V)$ is smooth and 10-dimensional.

Let $\mathbb{P}\left(\mathrm{H}^{0}\left(V, \mathcal{O}_{V}(1)\right)\right)=\mathbb{P}^{6}$. Notice that, once we fix the hyperplane section $S$, for any curve $C$ the intersection $C \cap S$ gives 5 points spanning $\mathbb{P}^{4} \subset \mathbb{P}^{6}$. Conversely, given any $\mathbb{P}^{4} \subset \mathbb{P}^{6}$, there is a curve $C$ such that the spaces $\langle C\rangle$ and $\langle S\rangle$ span $\mathbb{P}^{6}$. Fixing $S$ thus provides a birational map $\mathrm{H}_{5 t+1}(V)-\rightarrow \mathbb{G}\left(\mathbb{P}^{4}, \mathbb{P}^{6}\right)$.

Since $\operatorname{dim} \mathrm{H}_{5 t+1}(V)=\operatorname{dim} \operatorname{Hilb}_{5}\left(\mathbb{P}^{2}\right)=10$, we have to prove that the map $\zeta$ is generically finite. So we fix $\underline{e}=\left(e_{1}, \ldots, e_{5}\right)$ and consider the space $\mathbb{P}_{\underline{e}}^{4}=$ $\left\langle e_{1}, \ldots, e_{5}\right\rangle$. Varying a hyperplane section $S^{\prime}$ of $V$ in the pencil of hyperplanes containing $\mathbb{P}_{e}^{4}$, we obtain a ruled surface $S_{\underline{e}}^{j}$ consisting of exceptional lines in $S^{\prime}$ of type $b_{j}^{\prime}$. The ruled surface $S_{\underline{\underline{e}}}^{j}$ is not a cone, for there are finitely many lines through any point in $V$ (see [Iskovskikh and Prokhorov 1999, page 64] and [Furushima and Nakayama 1989]). Thus, its dual variety is a hypersurface in $\breve{\mathbb{P}}^{6}$.

Given a curve $C \subset S^{\prime}$, write $C=2 \ell-b_{1}^{\prime}$. We have $\zeta(C)=e_{1}+\cdots+e_{5}$ if and only if there is a hyperplane section $S^{\prime}=\mathbb{P}^{5} \cap V$ with $\mathbb{P}^{5} \supset \mathbb{P}_{e}^{4}$ and such that $\mathbb{P}^{5}$ contains the curve of class $2 \ell-b_{1}$. This happens if and only if the hyperplane $\mathbb{P}^{5}$ is tangent to the ruled surface $S_{e}^{1}$. Being the dual variety of the hypersurface $S_{e}^{1}$, it intersects the general pencil of $\mathbb{P}^{5}$ 's containing $\mathbb{P}_{\underline{b}}^{4}$ in a finite set of points.

Lemma 4.3. Let $S$ be a fixed hyperplane section and fix notation as in Definition 2.13. Define the linear systems

$$
\begin{aligned}
& \mathscr{L}_{9}=4 \ell-2 b_{1}-2 b_{2}-b_{3}-b_{4}-e_{1}-e_{2}-e_{3}-\sum f_{j}, \\
& \mathscr{L}_{10}=5 \ell-2 \sum b_{i}-2 e_{1}-e_{2}-e_{3}-\sum f_{j}, \\
& \mathscr{L}_{11}=4 \ell-2 b_{1}-2 b_{2}-b_{3}-b_{4}-e_{1}-e_{2}-\sum f_{j}, \\
& \mathscr{L}_{12}=5 \ell-2 \sum b_{i}-2 e_{1}-e_{2}-\sum f_{j}, \\
& \mathscr{L}_{13}=4 \ell-2 b_{1}-2 b_{2}-b_{3}-b_{4}-e_{1}-\sum f_{j} .
\end{aligned}
$$


Each $\mathscr{L}_{d}$ has positive dimension and contains a smooth element $\tilde{C}_{1}^{d}$. The curve $\varphi\left(\tilde{C}_{1}^{d}\right)$ is a smooth elliptic curve in $X$ of degree $d$, contained in precisely $14-d$ independent hyperplanes.

Proof. The linear systems $\mathscr{L}_{j}$ have positive dimension, as can be seen by counting parameters. Indeed, it suffices to compute the expected dimension of the linear system of curves in $\mathbb{P}^{2}$ passing through assigned points and with prescribed nodes.

For odd (even) $d$, the system $\mathscr{L}_{d}$ contains a smooth element $\tilde{C}_{1}^{d}$ if and only if there exists an irreducible plane quartic with nodes only at $B_{1}$ and $B_{2}$ (respectively, an irreducible plane quintic with nodes only at $B_{1}, \ldots, B_{6}$ and at the point in $\mathbb{P}^{2}$ corresponding to $e_{1}$ ). It suffices to project an elliptic normal quartic (quintic) in $\mathbb{P}^{3}\left(\mathbb{P}^{4}\right)$ from a general point (line) to obtain such a curve.

The degree of $\varphi\left(\tilde{C}_{1}^{d}\right)$ is easily computed to be $d=\mathscr{L}_{d} \cdot \mathscr{L}$, where $\mathscr{L}$ is the linear system of Definition 2.13.

Since any elliptic curve of degree $d \leq 13$ is contained in a hyperplane section $S_{22}$ of $X$, we have that

$$
\mathrm{h}^{0}\left(J_{C_{1}^{d}, X}(1)\right)=\mathrm{h}^{0}\left(J_{C_{1}^{d}, S_{22}}(1)\right)+1 .
$$

Using the map $\varphi$ and the fixed isomorphism $S \rightarrow \mathrm{Bl}_{B_{1}, \ldots B_{4}}\left(\mathbb{P}^{2}\right)$, we get

$$
\mathrm{h}^{0}\left(J_{C_{1}^{d}, S_{22}}(1)\right)=\mathrm{h}^{0}\left(\mathbb{P}^{2}, \mathscr{L}-\mathscr{L}_{d}\right) .
$$

It is then enough to compute the dimension of the following linear systems on $\mathbb{P}^{2}$ :

$$
\begin{aligned}
& \mathscr{L}-\mathscr{L}_{9}=5 \ell-b_{1}-b_{2}-2 b_{3}-2 b_{4}-e_{1}-e_{2}-e_{3}-2 e_{4}-2 e_{5}, \\
& \mathscr{L}-\mathscr{L}_{10}=4 \ell-\sum b_{i}-e_{2}-e_{3}-2 e_{4}-2 e_{5}, \\
& \mathscr{L}-\mathscr{L}_{11}=5 \ell-b_{1}-b_{2}-2 b_{3}-2 b_{4}-e_{1}-e_{2}-2 e_{3}-2 e_{4}-2 e_{5}, \\
& \mathscr{L}-\mathscr{L}_{12}=4 \ell-\sum b_{i}-e_{2}-2 e_{3}-2 e_{4}-2 e_{5}, \\
& \mathscr{L}-\mathscr{L}_{13}=5 \ell-b_{1}-b_{2}-2 b_{3}-2 b_{4}-e_{1}-2 e_{2}-2 e_{3}-2 e_{4}-2 e_{5} .
\end{aligned}
$$

Using Lemma 4.2, we can compute the dimension of these linear systems by choosing the points corresponding to the $e_{i}$ 's in a Zariski open set of $\mathrm{Hilb}_{5}\left(\mathbb{P}^{2}\right)$. Notice that $\operatorname{exp\operatorname {dim}}\left(\mathscr{L}-\mathscr{L}_{d}\right)=13-d$, so we need only check that expdim $\left(\mathscr{L}-\mathscr{L}_{d}\right)=$ $\operatorname{dim}\left(\mathscr{L}-\mathscr{L}_{d}\right)$. This we can do using Cremona transformations on $\mathbb{P}^{2}$.

For Case (13), consider the Cremona transformation $\gamma_{9}$ associated to the linear system $2 \ell-b_{3}-b_{4}-e_{4}$. Any curve in $\mathscr{L}-\mathscr{L}_{9}$ touches a conic through $b_{3}-$ $b_{4}-e_{4}$ in 4 points. Further, any curve in $\mathscr{L}-\mathscr{L}_{9}$ touches the line $\left\langle B_{3}, B_{4}\right\rangle$ (or, respectively, $\left\langle B_{4}, e_{4}\right\rangle$ or $\left\langle B_{3}, e_{4}\right\rangle$ ) in a single further point $e_{4}^{\prime}$ (or, respectively, $b_{3}^{\prime}$ or $\left.b_{4}^{\prime}\right)$. Therefore, the linear system $\mathscr{L}-\mathscr{L}_{9}$ is mapped under $\gamma_{9}$ to $4 \ell-b_{1}-$ $b_{2}-b_{3}^{\prime}-b_{4}^{\prime}-e_{1}-e_{2}-e_{3}-e_{4}^{\prime}-2 e_{5}$. By Lemma 4.2, the points $e_{1}, \ldots, e_{5}$ lie in general position. The points $b_{i}$ can be chosen generic, for we can define $S$ to 
be the blow-up of $\mathbb{P}^{2}$ at a general 4-tuple of points. Since we now have a linear system of plane quartics with only one node and passing through 8 general points, we conclude that $\mathrm{h}^{0}\left(\mathbb{P}^{2}, \mathscr{L}-\mathscr{L}_{9}\right)=4$.

In Case (15), define $\gamma_{11}$ as the Cremona transformation associated to $2 \ell-b_{3}-$ $b_{4}-e_{3}$, sending $\mathscr{L}-\mathscr{L}_{9}$ to $4 \ell-b_{1}-b_{2}-b_{3}^{\prime}-b_{4}^{\prime}-e_{1}-e_{2}-e_{3}^{\prime}-2 e_{4}-2 e_{5}$. Take $\gamma_{11}^{\prime}=\gamma_{2 \ell-e_{3}-e_{4}-b_{1}}$. Then $\gamma_{11}^{\prime} \circ \gamma_{11}$ sends $\mathscr{L}-\mathscr{L}_{9}$ to $3 \ell-b_{2}-b_{3}^{\prime}-b_{4}^{\prime}-e_{1}-e_{2}-$ $e_{3}^{\prime}-e_{4}^{\prime \prime}-e_{5}^{\prime \prime}$. And 8 general points impose 8 linearly independent conditions on the 10-dimensional space of plane cubics.

In Case (17), put $\gamma_{13}=\gamma_{2 \ell-b_{3}-b_{4}-e_{2}}$ and $\gamma_{13}^{\prime}=\gamma_{2 \ell-e_{3}-e_{4}-e_{5}}$. The linear system $\mathscr{L}-\mathscr{L}_{13}$ is mapped by $\gamma_{13}^{\prime} \circ \gamma_{13}$ to $2 \ell-b_{2}-b_{2}-b_{3}^{\prime}-b_{4}^{\prime}-e_{1}-e_{2}^{\prime}$. Since there is no conic through 6 general points, we are done.

In Case (14), set $\gamma_{10}=\gamma_{2 \ell-e_{3}-e_{4}-e_{5}}$. The lines $\left\langle e_{3}, e_{4}\right\rangle$ and $\left\langle e_{3}, e_{5}\right\rangle$ give rise to two extra points $e_{4}^{\prime}$ and $e_{5}^{\prime}$, so we compute $\mathrm{h}^{0}\left(3 \ell-\sum b_{i}-e_{2}-e_{4}^{\prime}-e_{5}^{\prime}\right)=3$.

In Case (16), put $\gamma_{12}=\gamma_{2 \ell-e_{3}-e_{4}-e_{5}}$. Here we have no extra points, and the statement follows since $\mathrm{h}^{0}\left(2 \ell-\sum b_{i}-e_{2}\right)=1$.

Proof of Proposition 4.1. The curve $C_{1}^{7}$ exists according to [Kuznetsov 1996; Faenzi 2006]. In fact, it is just the zero locus of a general global section $s$ from $\mathrm{H}^{0}\left(E^{*}\right) \simeq k^{8}$.

For $C_{1}^{8}$, consider a homomorphism $\alpha: K \rightarrow U$, with $\alpha \in \operatorname{Hom}(K, U) \simeq B^{*}$. This morphism is surjective whenever $\alpha$ lies outside the discriminant curve $\operatorname{det} \Psi^{\top} \subset$ $\mathbb{P}\left(B^{*}\right)$ (see Lemma 3.1), so for a general $\alpha$ we get a rank-2 locally free sheaf $F_{8}=\operatorname{ker} \alpha$. It follows easily from Lemma 2.12 that $c_{1}\left(F_{8}\right)=-1$ and $c_{2}\left(F_{8}\right)=8$. Taking global sections of $F_{8}^{*}$ and using the identifications of Lemma 2.7, we get

$$
\mathrm{H}^{0}\left(F_{8}^{*}\right) \simeq \operatorname{ker}\left(\alpha: \mathrm{S}^{4} B / F \rightarrow \mathrm{S}^{3} B / F\left(B^{*}\right)\right) .
$$

For a general $\alpha$, this map is surjective, so $\mathrm{h}^{0}\left(F_{8}^{*}\right)=7$. Further, $F_{8}^{*}$ is globally-generated since $K^{*}$ is. Therefore, a general section of $F_{8}^{*}$ vanishes along the required curve $C_{1}^{8}$.

Finally, for $9 \leq d \leq 13$ the statement follows from Lemma 4.3.

Proposition 4.4. On the general variety $X$, there exists a smooth elliptic curve $C_{1}^{d}$ of degree $d$ for $d=14$ or $d=15$. In both cases, $C_{1}^{d}$ is nondegenerate.

Proof. It is well-known that there exist smooth elliptic normal curves of degree 7 in $V$. Nonetheless, we sketch a quick proof.

Denote by $U_{V}$ and $Q_{V}$ the universal rank-2 subbundle and the universal rank3 quotient bundle on $\mathbb{G}\left(k^{2}, k^{5}\right)$, restricted to $V$. One proves that, for a general map $\alpha: U_{V}^{\oplus 2} \rightarrow\left(Q_{V}^{*}\right)^{\oplus 2}$, the sheaf coker $\alpha \otimes \mathcal{O}_{V}(1)$ is a globally-generated rank-2 bundle on $V$, whose general section vanishes on the required curve $D_{7}$.

Take now a hyperplane section $S$ and denote by $d_{1}, \ldots d_{7}$ the intersection points of $D_{7}$ with $S$. Recall the notation from Definition 2.13. Choose a smooth curve 
$C_{0}^{5}$ in the linear system $2 \ell-b_{1}-d_{1}-d_{2}-d_{3}$. Clearly, this linear system has positive dimension. The curve $D_{7}$ is mapped by $\varphi_{|\mathscr{L}|}$ to a smooth elliptic curve of degree 15 , for it intersects $C_{0}^{5}$ at 3 points with normal crossings. This curve is nondegenerate, since $D_{7}$ is nondegenerate as well.

Moving the hyperplane section $S$ in $\check{\mathbb{P}}^{6}$, we can suppose that the point $d_{4}$ coincides with the point $f_{1}$. Taking again $C_{0}^{5} \in\left|2 \ell-b_{1}-d_{1}-d_{2}-d_{3}\right|$, we have that $D_{7}$ is now mapped by $\varphi_{|\mathscr{L}|}$ to a nondegenerate smooth elliptic curve of degree 14; indeed, it intersects $C_{0}^{5}$ at 3 points and $T_{1}$ at 1 point, with normal crossings.

Proposition 4.5. Consider $d$ with $7 \leq d \leq 15$ and let $F_{d}$ be the rank-2 vector bundle over $X$ associated to the elliptic curve $C_{1}^{d}$ constructed above. We have $c_{1}\left(F_{d}\right)=-1$ and $c_{2}\left(F_{d}\right)=d$. Furthermore, $F_{d}$ is stable for any $d$, is $A C M$ when $7 \leq d \leq 14$, and has $\mathrm{h}^{0}\left(F_{15}^{*}\right)=\mathrm{h}^{1}\left(F_{15}^{*}\right)=1$ when $d=15$.

Proof. Set $C=C_{1}^{d}$. The numerical invariants of the bundle $F_{d}$ are obvious, while its stability follows at once from Hoppe's criterion.

By Serre duality and $(1)$, one has $\mathrm{h}^{2}\left(F_{d}^{*}\right)=\mathrm{h}^{1}\left(F_{d}(-1)\right)=\mathrm{h}^{1}\left(F_{d}^{*}(-2)\right)=0$.

Taking twisted sections in the sequence (1), we get that $F_{d}$ is ACM if and only if $\mathrm{h}^{1}\left(F_{d}(1)\right)=0$, that is, if and only if $\mathrm{h}^{1}\left(J_{C, X}(1)\right)=0$. Indeed, in this case the map $\mathrm{H}^{0}\left(\mathrm{O}_{X}(1)\right) \rightarrow \mathrm{H}^{0}\left(\mathrm{O}_{C}(1)\right)$ is surjective. This implies that $\mathrm{H}^{0}\left(\mathrm{O}_{X}(t)\right) \rightarrow \mathrm{H}^{0}\left({ }{ }_{C}(t)\right)$ is surjective for all $t \geq 1$, so $\mathrm{h}^{1}\left(J_{C, X}(t)\right)=0$ for $t \geq 1$. After using (1), we get $\mathrm{h}^{1}\left(F_{d}(t)\right)=0$ for $t \geq 1$. For $t \leq 0$ this trivially holds as well, so, by Serre duality, $F_{d}$ is ACM.

This happens precisely when $\mathrm{h}^{0}\left(J_{C, X}(1)\right)=14-d$, so the conclusion follows from Propositions 4.1 and 4.4.

Theorem 4.6. For $d$ with $8 \leq d \leq 15$, the bundle $F_{d}$ of Proposition 4.5 is isomorphic to the cohomology of a monad

$$
E^{\oplus d-8} \stackrel{\beta_{d}}{\longrightarrow} K^{\oplus d-7} \stackrel{\alpha_{d}}{\longrightarrow} U^{\oplus d-7} .
$$

For $d=7$, the bundle $F_{7}$ is isomorphic to $E$.

Proof. From Hirzebruch-Riemann-Roch we get the equalities

$$
\begin{aligned}
& \chi\left(Q^{*} \otimes F_{d}\right)=d-7, \\
& \chi\left(U \otimes F_{d}\right)=d-7, \\
& \chi\left(E \otimes F_{d}\right)=d-8 .
\end{aligned}
$$

Recall that the vector bundles $U, Q^{*}, E$ and $F_{d}$ are stable. Hence, by [Maruyama 1981, Theorem 1.14], any tensor product between them is also stable. This implies at once the vanishings

$$
\mathrm{h}^{0}\left(Q^{*} \otimes F_{d}\right)=0, \quad \mathrm{~h}^{0}\left(U \otimes F_{d}\right)=0, \quad \mathrm{~h}^{0}\left(E \otimes F_{d}\right)=0 .
$$


Serre duality also yields

$$
\begin{array}{ll}
\mathrm{h}^{3}\left(Q^{*} \otimes F_{d}\right)=\mathrm{h}^{0}\left(Q \otimes F_{d}\right)=0 & \text { because } \mu\left(Q \otimes F_{d}\right)=-1 / 4, \\
\mathrm{~h}^{3}\left(U \otimes F_{d}\right)=\mathrm{h}^{0}\left(U^{*} \otimes F_{d}\right)=0 & \text { because } \mu\left(U^{*} \otimes F_{d}\right)=-1 / 6, \\
\mathrm{~h}^{3}\left(E \otimes F_{d}\right)=\mathrm{h}^{0}\left(E^{*} \otimes F_{d}\right)=0 & \text { because } c_{2}(E) \neq c_{2}\left(F_{d}\right) .
\end{array}
$$

Here, (21) follows since $\mu(E)=\mu\left(F_{d}\right)=-1 / 2$, but $c_{2}(E)=7 \neq d=c_{2}\left(F_{d}\right)$, so $\operatorname{Hom}\left(E, F_{d}\right)=0$.

Consider the tensor product of the bundle $F_{d}$ by the sequences (6), (9), and the dual of sequence (6). Since $\mathrm{h}^{0}\left(F_{d}\right)=0$ and $\mathrm{h}^{1}\left(F_{d}\right)=0$, we have

$$
\begin{array}{ll}
\mathrm{h}^{1}\left(Q^{*} \otimes F_{d}\right)=\mathrm{h}^{0}\left(U^{*} \otimes F_{d}\right)=0 & \text { by }(20), \\
\mathrm{h}^{1}\left(U \otimes F_{d}\right)=\mathrm{h}^{0}\left(Q \otimes F_{d}\right)=0 & \text { by }(19), \\
\mathrm{h}^{1}\left(E \otimes F_{d}\right)=\mathrm{h}^{0}\left(\left(E^{\prime}\right)^{*} \otimes F_{d}\right) . &
\end{array}
$$

The group $\mathrm{H}^{0}\left(\left(E^{\prime}\right)^{*} \otimes F_{d}\right)$ vanishes as well, because $E^{\prime}$ is a stable bundle as well, and we have $\mu\left(\left(E^{\prime}\right)^{*} \otimes F_{d}\right)=-1 / 3$. Summing up:

$$
\mathrm{h}^{2}\left(Q^{*} \otimes F_{d}\right)=d-7, \quad \mathrm{~h}^{2}\left(U \otimes F_{d}\right)=d-7, \quad \mathrm{~h}^{2}\left(E \otimes F_{d}\right)=d-8 .
$$

This implies that $F_{d}$ is isomorphic to the cohomology of a monad of form (18). Clearly, for $d=7$ the above argument implies $E \simeq F_{7}$.

Theorem 4.7. Consider $d$ with $7 \leq d \leq 15$, and let $X$ be general. Take the Hilbert scheme $\mathscr{H}_{d, 1}(X)$ of curves in $X$ of degree $d$ and arithmetic genus 1 . At generic points, $\mathscr{H}_{d, 1}(X)$ is smooth of dimension $d$ and the moduli space $\mathrm{M}_{X}(2 ;-1, d)$ is smooth of dimension $2 d-14$.

Proof. Let $Z=C_{1}^{d}$ be a curve of degree $d$ and arithmetic genus 1, contained in $X$. Consider the vector bundle $F_{d}$ associated to $Z$.

Tensoring by $F_{d}$ both the exact sequence (1) and the exact sequence defining $Z \subset X$, we get, after using the isomorphism (2), the exact sequences

$$
\begin{aligned}
& 0 \longrightarrow F_{d} \longrightarrow \mathscr{E} n d\left(F_{d}\right) \longrightarrow F_{d}^{*} \otimes J_{Z, X} \longrightarrow 0, \\
& 0 \longrightarrow F_{d}^{*} \otimes J_{Z, X} \longrightarrow F_{d}^{*} \longrightarrow N_{Z, X} \longrightarrow 0 .
\end{aligned}
$$

Taking global sections, we get $\mathrm{h}^{2}\left(X, \mathscr{E} n d\left(F_{d}\right)\right)=\mathrm{h}^{1}\left(Z, N_{Z, X}\right)$. This means that $\mathrm{M}_{X}(2 ;-1, d)$ is unobstructed at $\left[F_{d}\right]$ if and only if $\mathscr{H}_{d, 1}(X)$ is unobstructed at $[Z]$.

Consider now the monad (18) given by Theorem 4.6. Denote by $W_{d}^{1}$ the vector space $\mathrm{H}^{2}\left(Q^{*} \otimes F_{d}\right) \simeq k^{d-7}$, and by $W_{d}^{2}$ the space $\mathrm{H}^{2}\left(U \otimes F_{d}\right) \simeq k^{d-7}$. An element $(m, n)$ of the group $\operatorname{SL}\left(W_{d}^{1}\right) \times \operatorname{SL}\left(W_{d}^{2}\right)$ acts on $\mathbb{P}\left(\operatorname{Hom}(K, U) \otimes \operatorname{Hom}\left(W_{d}^{1}, W_{d}^{2}\right)\right)$ by taking $\alpha_{d}$ to $n \circ \alpha_{d} \circ m^{-1}$. For a general $\alpha_{d}$, this action is free. Taking now the 
functor $\operatorname{Hom}(E,-)$, we get a morphism:

$$
\operatorname{Hom}(K, U) \otimes \operatorname{Hom}\left(W_{d}^{1}, W_{d}^{2}\right) \longrightarrow A^{*} \otimes A^{*} \otimes \operatorname{Hom}\left(W_{d}^{1}, W_{d}^{2}\right) .
$$

Recall from (5) that $\operatorname{Hom}(K, U) \simeq B^{*}$. Hence, any element $\alpha_{d}$ in the vector space $\operatorname{Hom}(K, U) \otimes \operatorname{Hom}\left(W_{d}^{1}, W_{d}^{2}\right)$ can be seen as a map $W_{d}^{1} \rightarrow W_{d}^{2}$ with entries in $B^{*}$. The morphism (24) takes the map $\alpha_{d}$ to a $4(d-7) \times 4(d-7)$ square matrix $W_{d}^{1} \otimes A \rightarrow W_{d}^{2} \otimes A^{*}$, whose entries are given by $\Psi^{\top} \otimes \mathrm{id}_{\left(W_{d}^{1}\right)^{*}} \otimes \mathrm{id}_{W_{d}^{2}}$. Denote this matrix by $\Psi^{\top}\left(\alpha_{d}\right)$ (see Lemma 3.1).

Consider the sheaf $\operatorname{ker}\left(\alpha_{d}: W_{d}^{1} \otimes K \rightarrow W_{d}^{2} \otimes U\right)$. The above discussion implies that there exists an injective map $\beta_{d}: E^{d-8} \hookrightarrow \operatorname{ker} \alpha_{d}$ if and only if $\operatorname{rk} \Psi^{\top}\left(\alpha_{d}\right) \leq$ $4(d-7)-(d-8)=3 d-20$. Since $F_{d}$ is stable and $\mathrm{h}^{2}\left(E \otimes F_{d}\right)=d-8$, there is a unique $\beta_{d}$ up to isomorphisms.

Summing up, around $\left[F_{d}\right]$ there exists an open neighborhood of an irreducible component of the moduli space $\mathrm{M}_{X}(2 ;-1, d)$, isomorphic to the set

$$
\begin{aligned}
\mathrm{M}(d)=\left\{\left[\alpha_{d}\right] \in \mathbb{P}\left(B^{*} \otimes \operatorname{Hom}\left(W_{d}^{1}, W_{d}^{2}\right)\right) \mid\right. & \operatorname{rk} \Psi^{\top}\left(\alpha_{d}\right)= \\
& 3 d-20\} \\
& / \operatorname{SL}(d-7) \times \operatorname{SL}(d-7) .
\end{aligned}
$$

For a sufficiently general $\Psi^{\top}: B^{*} \rightarrow A^{*} \otimes A^{*}$, the variety $\mathrm{M}(d)$ admits smooth points, indeed it is obtained by cutting the smooth subset of the variety of $(3 d-20)$ secant $(3 d-19)$-spaces to the Segre image of $\mathbb{P}^{4 d-27} \times \mathbb{P}^{4 d-27}$ by a sufficiently general linear space.

It is easy to check that the dimension of $\mathrm{M}(d)$ at a smooth point $\left[\alpha_{d}^{\prime}\right]$ is $2 d-14$. At the bundle $\left[F_{d}^{\prime}\right]$ corresponding to $\left[\alpha_{d}^{\prime}\right]$, the dimension of $\mathrm{M}_{X}(2 ;-1, d)$ is also $2 d-14$. Thus, taking a section of the general bundle $F_{d}^{\prime}$, we obtain a curve $(Z)^{\prime}$ with $\mathrm{h}^{1}\left(N_{(Z)^{\prime}, X}\right)=0$, so $\mathrm{h}^{0}\left(N_{(Z)^{\prime}, X}\right)=d$. Therefore, the Hilbert scheme $\mathscr{H}_{d, 1}(X)$ is $d$-dimensional and smooth at $\left[(Z)^{\prime}\right]$.

End of the proof of Lemma 2.16. Consider a general hyperplane section $S_{22}$ of $X$. It is a K3 surface of Picard number $\rho\left(S_{22}\right)=1$. Take $F_{d}$ as defined in Proposition 4.5. Restricting $F_{d}$ to $S_{22}$, we get a stable rank-2 vector bundle on $S_{22}$. The moduli space $\mathrm{M}_{S_{22}}(2 ;-1, d)$ is then smooth and projective, of dimension $-\chi\left(\operatorname{End}\left(S_{22}, F_{d}\right)\right)-2$. It is immediate to check that $\operatorname{dim} \mathrm{M}_{S_{22}}(2 ;-1, d)=4 d-28$. Hence $d \geq 7$.

\section{Canonical and half-canonical curves}

We now prove the existence of the bundles from Cases (4) and (5) of Lemma 2.16. We deal with the latter case first.

Half-canonical curves. We prove the existence of a smooth half-canonical curve $C_{60}^{59}$ by a deformation argument. 
Lemma 5.1. There exists a smooth curve $Z=C_{60}^{59}$ in $X$ of degree 59 and genus 60 , given as the zero locus of a section of an ACM vector bundle $\mathscr{F}_{-1,15}(2)$. We have $\left.\omega_{Z} \simeq \mathcal{O}_{X}(2)\right|_{Z}$. The ACM bundle $\mathscr{F}_{-1,15}$ specializes to the non-ACM bundle $F_{15}$.

Proof. Recall from Proposition 4.4 that there exists an elliptic curve $C=C_{1}^{15}$ such that $\mathrm{h}^{1}\left(J_{C, X}\right)=1$ and $C$ is not contained in any hyperplane. According to Proposition 4.5, the vector bundle $F_{15}^{*}$ has a unique section vanishing along $C$.

By Theorem 4.6, the moduli space $\mathrm{M}_{X}(2 ;-1,15)$ is smooth and 16-dimensional at a general $\left[F_{15}\right]$. Consider the irreducible component of $\mathrm{M}_{X}(2 ;-1,15)$ that contains $\left[F_{15}\right]$ and take an open neighborhood of $\left[F_{15}\right]$ contained in this component. Pick a point $\left[F_{15}^{\prime}\right]$ belonging to this neighborhood and represented by a stable bundle $F_{15}^{\prime}$ not isomorphic to $F_{15}$.

Suppose $F_{15}^{\prime}(1)$ has a nontrivial global section $s$. Recall that $\mathrm{h}^{0}\left(F_{15}\right)=0$ by stability. The zero locus of $s$ would be a curve $C^{\prime}$ of degree 15 and arithmetic genus 1 . Therefore, $s$ would give a point $\left[C^{\prime}\right]$ in $\mathscr{H}_{15,1}(X)$. The point $\left[C^{\prime}\right]$ could not coincide with $[C]$, for otherwise $J_{C^{\prime}, X} \simeq J_{C, X}$ would yield $F_{15}^{\prime} \simeq F_{15}$.

Since $\mathscr{H}_{15,1}(X)$ is smooth of dimension 15 at $[C]$, the above discussion proves that the map $\tau: \mathscr{H}_{15,1}(X) \rightarrow \mathrm{M}_{X}(2 ;-1,15)$ is an open embedding at $[C]$, and that its image is the codimension- 1 locus $\left\{\left[F_{15}^{\prime}\right] \in \mathrm{M}_{X}(2 ;-1,15) \mid \mathrm{h}^{0}\left(F_{15}^{\prime}(1)\right) \neq 0\right\}$. Thus, for a general $\left[F_{15}^{\prime}\right]$ we must have $\mathrm{h}^{0}\left(F_{15}^{\prime}(1)\right)=0$.

Now, since $\chi\left(F_{15}^{\prime}(1)\right)=0$, we also get $\mathrm{h}^{1}\left(F_{15}^{\prime}(1)\right)=0$. We set $\mathscr{F}_{-1,15}=F_{15}^{\prime}$, and then $\mathscr{F}_{-1,15}$ is ACM. Finally, by Castelnuovo-Mumford regularity, $\mathscr{F}_{-1,15}(2)$ is globally generated, so a general section vanishes along a smooth curve $Z$ with the required invariants.

Remark 5.2. Any ACM stable bundle of type $\mathscr{F}_{-1,15}$ is the cohomology of a monad of type (18) with $d=15$. Indeed, it suffices to apply the proof of Theorem 4.6 to $\mathscr{F}_{-1,15}$.

Canonical curves. Here we will prove the existence of a smooth canonical curve in $X$ by exhibiting the bundle $\mathscr{F}_{0,4}$ of Lemma 2.16 .

Lemma 5.3. Given a general homomorphism $\alpha: U^{\oplus 2} \rightarrow\left(Q^{*}\right)^{\oplus 2}$, the sheaf coker $\alpha$ is a vector bundle of type $\mathscr{F}_{0,4}$.

Proof. Let $W_{1}$ and $W_{2}$ be 2-dimensional vector spaces such that the domain and codomain of $\alpha$ are $W_{1} \otimes U$ and $W_{2} \otimes Q^{*}$. Let $p_{1}: k \rightarrow W_{1}$ be an element of $\check{\mathbb{P}}\left(W_{1}\right)$ and $p_{2}: W_{2} \rightarrow k$ an element of $\mathbb{P}\left(W_{2}\right)$. To the pair $\left(p_{1}, p_{2}\right)$ we associate a map $U \rightarrow Q^{*}$ via the morphism

$$
\eta_{\alpha}: \mathbb{P}^{1} \times \mathbb{P}^{1} \rightarrow \mathbb{P}^{2}=\mathbb{P}(B), \quad\left(p_{1}, p_{2}\right) \mapsto\left(p_{2} \otimes \mathrm{id}_{Q^{*}}\right) \circ \alpha \circ\left(p_{1} \otimes \mathrm{id}_{U^{*}}\right) .
$$

For a general $\alpha$, the map $\eta_{\alpha}$ is a 2:1 cover. Suppose now that $\alpha$ is not injective, as a bundle map, at some given point $x$ of $X$. Then there exists $p_{1}: k \rightarrow W_{1}$ such that, 
for any $p_{2}: W_{2} \rightarrow k$, the map $\eta_{\alpha}\left(p_{1}, p_{2}\right)$ is zero over $x$. Equivalently, $x$ lies in the conic whose ideal is coker $\eta_{\alpha}\left(p_{1}, p_{2}\right)$. Since $\eta_{\alpha}$ is a finite map, this means that $x$ lies in the pencil of conics parameterized by $p_{2} \in \mathbb{P}\left(W_{2}\right)$, thus contradicting Lemma 3.2. Therefore coker $\alpha$ is locally free and, by a straightforward computation, it has the required Chern classes.

From the exact sequence $0 \longrightarrow U^{\oplus 2} \longrightarrow\left(Q^{*}\right)^{\oplus 2} \longrightarrow \mathscr{F}_{0,4} \longrightarrow 0$, we see immediately that $\mathrm{h}^{0}\left(\mathscr{F}_{0,4}\right)=0$ and $\mathrm{h}^{1}\left(\mathscr{F}_{0,4}(t)\right)=0$ for any $t \in \mathbb{Z}$; indeed, $U$ and $Q^{*}$ are ACM bundles.

Therefore $\mathscr{F}_{0,4}$ is stable and ACM. Indeed, Serre duality gives $\mathrm{h}^{2}\left(\mathscr{F}_{0,4}(t)\right)=$ $\mathrm{h}^{1}\left(\mathscr{F}_{0,4}(-1-t)\right)=0$ for all $t \in \mathbb{Z}$. Finally, one can compute

$$
\mathrm{h}^{1}\left(Q^{*} \otimes \mathscr{F}_{0,4}(1)\right)=0, \quad \mathrm{~h}^{2}\left(U \otimes \mathscr{F}_{0,4}(1)\right)=0, \quad \mathrm{~h}^{3}\left(E \otimes \mathscr{F}_{0,4}(1)\right)=0 .
$$

By Corollary 2.15 , we get that $\mathscr{F}_{0,4}(1)$ is globally generated, hence the zero locus of its general global section is the required canonical curve.

Lemma 5.4. Any ACM stable vector bundle of type $\mathscr{F}_{0,4}$ is the cokernel of a map $\alpha: U^{\oplus 2} \rightarrow\left(Q^{*}\right)^{\oplus 2}$.

Proof. The argument is analogous to that of Theorem 4.6. We find $\mathrm{h}^{p}\left(U \otimes \mathscr{F}_{0,4}\right)=$ 0 for $p \neq 1, \mathrm{~h}^{p}\left(K \otimes \mathscr{F}_{0,4}\right)=0$ for $p \neq 1$, and $\mathrm{h}^{p}\left(E \otimes \mathscr{F}_{0,4}\right)=0$ for all $p$. We conclude that $\mathrm{h}^{1}\left(U \otimes \mathscr{F}_{0,4}\right)=-\chi\left(U \otimes \mathscr{F}_{0,4}\right)=2$ and that $\mathrm{h}^{1}\left(K \otimes \mathscr{F}_{0,4}\right)=$ $-\chi\left(K \otimes \mathscr{F}_{0,4}\right)=2$, so the statement follows from Lemma 2.14 .

Remark 5.5. Summing up, we found that an open subset of a component of $\mathrm{M}_{X}(2 ; 0,4)$ is isomorphic to an open subset of the variety of Kronecker modules

$$
\mathbb{P}\left(W_{1}^{*} \otimes W_{2} \otimes B\right) / \mathrm{SL}\left(W_{1}\right) \times \mathrm{SL}\left(W_{2}\right),
$$

where $W_{1}$ and $W_{2}$ are 2-dimensional vector spaces. In particular, it is unirational and generically smooth of dimension 5 .

\section{Acknowledgments}

Both authors thank the Department of Mathematics of the Università di Firenze and of the Universidad Complutense de Madrid for the friendly hospitality and the opportunity to work together on several occasions.

Many computations involved in the present paper were considerably aided by the Maple package Schubert, developed by Sheldon Katz, Stein Arild Strømme, and Jan-Magnus Økland; see [Katz and Strømme 1992].

\section{References}

[Arrondo and Costa 2000] E. Arrondo and L. Costa, "Vector bundles on Fano 3-folds without intermediate cohomology", Comm. Algebra 28:8 (2000), 3899-3911. MR 2001d:14043 Zbl 1004.14010 
[Arrondo and Graña 1999] E. Arrondo and B. Graña, "Vector bundles on $G(1,4)$ without intermediate cohomology", J. Algebra 214:1 (1999), 128-142. MR 2000e:14069 Zbl 0963.14027

[Buchweitz et al. 1987] R.-O. Buchweitz, G.-M. Greuel, and F.-O. Schreyer, "Cohen-Macaulay modules on hypersurface singularities. II", Invent. Math. 88:1 (1987), 165-182. MR 88d:14005 Zbl 0617.14034

[Drezet 1986] J.-M. Drezet, "Fibrés exceptionnels et suite spectrale de Beilinson généralisée sur $\mathbf{P}_{2}$ (C)", Math. Ann. 275:1 (1986), 25-48. MR 88b:14014 Zbl 0578.14013

[Ellingsrud et al. 1987] G. Ellingsrud, R. Piene, and S. A. Strømme, "On the variety of nets of quadrics defining twisted cubics", pp. 84-96 in Space curves (Rocca di Papa, 1985), Lecture Notes in Math. 1266, Springer, Berlin, 1987. MR 88h:14034 Zbl 0659.14027

[Faenzi 2005] D. Faenzi, "Bundles over the Fano threefold V5", Comm. Algebra 33:9 (2005), 30613080. MR 2006g:14067 Zbl 1082.14044

[Faenzi 2006] D. Faenzi, "Bundles over Fano threefolds of type $V_{22}$ ", Ann. Mat. Pura Appl. (2006). To appear.

[Furushima and Nakayama 1989] M. Furushima and N. Nakayama, "The family of lines on the Fano threefold $V_{5}$ ”, Nagoya Math. J. 116 (1989), 111-122. MR 90k:14039 Zbl 0731.14025

[Gorodentsev 1990] A. L. Gorodentsev, "Exceptional objects and mutations in derived categories", pp. 57-73 in Helices and vector bundles, London Math. Soc. Lecture Note Ser. 148, Cambridge Univ. Press, Cambridge, 1990. MR 91m:14024 Zbl 0722.14007

[Hartshorne 1974] R. Hartshorne, "Varieties of small codimension in projective space", Bull. Amer. Math. Soc. 80 (1974), 1017-1032. MR 52 \#5688 Zbl 0304.14005

[Horrocks 1964] G. Horrocks, "Vector bundles on the punctured spectrum of a local ring", Proc. London Math. Soc. (3) 14 (1964), 689-713. MR 30 \#120 Zbl 0126.16801

[Huybrechts and Lehn 1997] D. Huybrechts and M. Lehn, The geometry of moduli spaces of sheaves, Aspects of Mathematics, E31, Friedr. Vieweg \& Sohn, Braunschweig, 1997. MR 98g:14012 Zbl 0872.14002

[Iskovskih 1978] V. A. Iskovskih, "Fano threefolds, II”, Izv. Akad. Nauk SSSR Ser. Mat. 42:3 (1978), 506-549. In Russian; translated in Math. USSR Izv. 12:3 (1978), 469-506. MR 80c:14023b

[Iskovskikh 1989] V. A. Iskovskikh, "Double projection from a line onto Fano 3-folds of the first kind", Mat. Sb. 180:2 (1989), 260-278. In Russian; translated in Math. USSR Sb. 66:1 (1990), 265-284. MR 90j:14048 Zbl 0691.14027

[Iskovskikh and Prokhorov 1999] V. A. Iskovskikh and Y. G. Prokhorov, "Fano varieties", pp. 1-247 in Algebraic geometry, vol. V, Encyclopaedia Math. Sciences 47, Springer, Berlin, 1999. MR 2000b:14051b Zbl 0912.14013

[Katz and Strømme 1992] S. Katz and S. A. Strømme, "Schubert, a Maple package for intersection theory and enumerative geometry", 1992, Available at http://www.mi.uib.no/stromme/schubert/.

[Knörrer 1987] H. Knörrer, "Cohen-Macaulay modules on hypersurface singularities, I", Invent. Math. 88:1 (1987), 153-164. MR 88d:14004 Zbl 0617.14033

[Kuznetsov 1996] A. G. Kuznetsov, "An exception set of vector bundles on the varieties V22", Vestnik Moskov. Univ. Ser. I Mat. Mekh. 3 (1996), 41-44, 92. In Russian; translation in Moscow Univ. Math. Bull. 51:3 (1996), 35-37. MR 97m:14040 Zbl 0913.14010

[Madonna 2000] C. Madonna, "Rank-two vector bundles on general quartic hypersurfaces in $\mathbb{P}^{4}$ ", Rev. Mat. Complut. 13:2 (2000), 287-301. MR 2002d:14064 Zbl 0981.14019

[Madonna 2002] C. G. Madonna, "ACM vector bundles on prime Fano threefolds and complete intersection Calabi-Yau threefolds", Rev. Roumaine Math. Pures Appl. 47:2 (2002), 211-222 (2003). MR 2004g:14048 Zbl 1051.14050

[Maruyama 1981] M. Maruyama, "The theorem of Grauert-Mülich-Spindler", Math. Ann. 255:3 (1981), 317-333. MR 82k:14012 Zbl 0438.14015 
[Mukai 1992] S. Mukai, "Fano 3-folds", pp. 255-263 in Complex projective geometry (Trieste and 1989 / Bergen, 1989), edited by G. Ellingsrud et al., London Math. Soc. Lecture Note Ser. 179, Cambridge Univ. Press, Cambridge, 1992. MR 94a:14042 Zbl 0774.14037

[Mukai 2004] S. Mukai, "Plane quartics and Fano threefolds of genus twelve", pp. 563-572 in The Fano Conference (Torino, 2002), edited by A. Collino et al., Università di Torino, Dipartimento di Matematica, Turin, 2004, Available at http://www.kurims.kyoto-u.ac.jp/home_page/preprint/PS/ RIMS1422.ps.gz. MR 2006d:14043 Zbl 1068.14050

[Okonek et al. 1980] C. Okonek, M. Schneider, and H. Spindler, Vector bundles on complex projective spaces, Progress in Mathematics 3, Birkhäuser, Mass., 1980. MR 81b:14001 Zbl 0438.32016 [Ottaviani 1987] G. Ottaviani, "Critères de scindage pour les fibrés vectoriels sur les Grassmanniennes et les quadriques”, C. R. Acad. Sci. Paris Sér. I Math. 305:6 (1987), 257-260. MR 88j:14024 Zbl 0629.14012

[Ottaviani 1989] G. Ottaviani, "Some extensions of Horrocks criterion to vector bundles on Grassmannians and quadrics", Ann. Matematica Pura Appl. (4) 155 (1989), 317-341. MR 91f:14018 Zbl 0718.14010

[Rudakov 1990] A. N. Rudakov, "Exceptional collections, mutations and helices", pp. 1-6 in Helices and vector bundles, London Math. Soc. Lecture Note Ser. 148, Cambridge Univ. Press, Cambridge, 1990. Seminaire Rudakov, Translated from the Russian by A. D. King, P. Kobak and A. Maciocia. MR 93a:14016 Zbl 0721.14011

[Schreyer 2001] F.-O. Schreyer, "Geometry and algebra of prime Fano 3-folds of genus 12", Compositio Math. 127:3 (2001), 297-319. MR 2002d:14062 Zbl 1049.14036

[Serre 1963] J.-P. Serre, "Sur les modules projectifs", in Séminaire d'algèbre et de théorie de nombres 14 (Séminaire P. Dubreil), 1960/1961, Sécrétariat mathématique, Paris, 1963. Exp. no. 2. Zbl 0132.41301

[Takeuchi 1989] K. Takeuchi, "Some birational maps of Fano 3-folds", Compositio Math. 71:3 (1989), 265-283. MR 90k:14041 Zbl 0712.14025

[Vogelaar 1978] J. A. Vogelaar, Constructing vector bundles from codimension-two subvarieties, Ph.D. thesis, Univ. Leiden, 1978.

Received September 12, 2004. Revised March 7, 2005.

\author{
ENRIQUE ARRONDO \\ DEPARTAMENTo DE Álgebra \\ FACUlTAd de Ciencias Matemáticas \\ UNIVERSIDAD COMPLUTENSE DE MADRID \\ 28040 MADRID \\ SPAIN \\ Enrique_Arrondo@mat.ucm.es \\ http://www.mat.ucm.es/ arrondo \\ DANIELE FAENZI \\ Dipartimento di MATEMATICA "U. Dini" \\ UNIVERSITÀ DI FIRENZE \\ Viale Morgagni 67/A \\ I-50134, FLORENCE \\ ITALY \\ faenzi@math.unifi.it \\ http://www.math.unifi.it/ faenzi/
}

